\title{
Sonographic assessment of lower uterine segment at term with previous caesarean section
}

\author{
Sandip Lahiri, Suresh Chandra Mondal*, Sudip Ranjan Pal
}

Department of Obstetrics \& Gynaecology, Malda Medical College and Hospital, Malda West Bengal, India

Received: 14 December 2014

Accepted: 03 January 2015

*Correspondence:

Dr. Suresh Chandra Mondal,

E-mail: sureshmondal@gmail.com,sureshmondal77@gmail.com

Copyright: (C) the author(s), publisher and licensee Medip Academy. This is an open-access article distributed under the terms of the Creative Commons Attribution Non-Commercial License, which permits unrestricted non-commercial use, distribution, and reproduction in any medium, provided the original work is properly cited.

\begin{abstract}
Background: The present study is an attempt to evaluate the efficacy of trans abdominal sonography to measure the thickness of scarred lower uterine segment at term and to detect anatomical defect if there is any to predict the risk of scar rupture.

Methods: This study assessed the usefulness of sonographic measurement of lower uterine segment thickness in 100 patients with previous Caesarean patients. Lower uterine segment thickness was compared by antenatal sonography caliper measurements and observations during caesarean section.

Results: Lower uterine segment thickness measured by ultrasonography correlated well with the thickness measured by vernier calipers at Caesarean section. Sensitivity of $92.86 \%$, specificity of $77.27 \%$, positive predictive value of $83.87 \%$ and negative predictive value of $89.47 \%$ suggested that if the thickness of lower uterine segment was $2.6 \mathrm{~mm}$ or more, chances of vaginal delivery following trial of labour was high.

Conclusions: Antenatal ultrasonographic assessment of lower uterine segment thickness can result a successful trial of labour in women with previous caesarean section.
\end{abstract}

Keywords: Sonography, Lower uterine segment, Previous L.S.C.S.

\section{INTRODUCTION}

The old dictum 'Once a caesarean section, always a caesarean section' has been subjected to critical analysis by the obstetric world. To put a check on this trend, a change of policy in favour of vaginal delivery after previous caesarean section is needed. Several methods have been used to evaluate the lower uterine segment after caesarean section. Sonographic methods can be used to evaluate the lower uterine segment thickness. If a technique could be developed to predict the integrity of scarred uterus before labour, a large proportion of patients would be considered for a trial of labour in future protocol. The purpose of this study is to assess usefulness of sonographic measurement of lower uterine segment thickness at term and to identify defective lower uterine segment in women at term with previous Caesarean section.

\section{METHODS}

This prospective study to assess the usefulness of sonographic measurement of lower uterine segment thickness in previous caesarean patients was carried out at Malda medical college during the period from September 2013 to August 2014. During this period, a total of 100 patients with history of previous Caesarean section admitted in the hospital were assessed.

Out of a total of 100 patients, 50 patients (50\%) were subjected to elective repeat Caesarean section, either because of recurrent, or a new indication. The remaining 50 cases $(50 \%)$ were given trial of labour.

Measurement of lower uterine segment thickness was made by the following ways: 


\section{Antenatal sonography}

The thickness of the anterior wall of lower uterine segment, where it covers the foetal pole, was measured from the interface of the urine and posterior wall of the bladder to the interface of amniotic fluid and decidua. Each study included multiple measurements of the thickness of anterior wall of lower uterine segment, both in longitudinal and transverse planes. The lowest value measured was used to describe the thickness of lower uterine segment.

\section{Observations at caesarean section}

At caesarean section, the thickness of lower uterine segment was measured using vernier calipers in the centre and at two lateral angles after delivery of placenta.

\section{RESULTS}

Table 1 shows correlation of lower uterine segment thickness by ultrasonography and calipers.

Table 1: Correlation of lower uterine segment thickness by ultrasonography and calipers.

\begin{tabular}{|c|c|c|c|}
\hline $\begin{array}{l}\text { Route of } \\
\text { present } \\
\text { delivery in } \\
\text { patients } \\
\text { with } \\
\text { previous } \\
\text { caesarean } \\
\text { section }\end{array}$ & $\begin{array}{l}\text { Lower } \\
\text { uterine } \\
\text { segment } \\
\text { thickness by } \\
\text { USG (mm) } \\
\text { Mean } \pm \text { SD }\end{array}$ & $\begin{array}{l}\text { Lower } \\
\text { uterine } \\
\text { segment } \\
\text { thickness by } \\
\text { calipers } \\
(\mathrm{mm}) \\
\text { Mean } \pm \text { SD }\end{array}$ & $\begin{array}{l}\text { Statistical } \\
\text { significance }\end{array}$ \\
\hline $\begin{array}{l}\text { Elective } \\
\text { caesarean } \\
\mathrm{N}=50\end{array}$ & $2.67 \pm 0.192)$ & $2.56 \pm 0.225$ & $\begin{array}{l}\mathrm{t}=2.63 \\
\mathrm{P}<0.05\end{array}$ \\
\hline $\begin{array}{l}\text { Emergency } \\
\text { caesarean } \\
\mathrm{N}=28\end{array}$ & $2.48 \pm 0.153$ & $2.44 \pm 0.174$ & $\begin{array}{l}\mathrm{t}=0.91 \\
\mathrm{P}>0.1\end{array}$ \\
\hline $\begin{array}{l}\text { All cases of } \\
\text { caesarean } \\
\mathrm{N}=78\end{array}$ & $2.60 \pm 0.198$ & $2.52 \pm 0.215$ & $\begin{array}{l}\mathrm{t}=2.42 \\
\mathrm{P}<0.05\end{array}$ \\
\hline $\begin{array}{l}\text { Normal } \\
\text { delivery } \\
\mathrm{N}=22\end{array}$ & $2.62 \pm 0.067$ & & \\
\hline
\end{tabular}

Table 2: Correlation between type of previous caesarean section and scar thickness.

\begin{tabular}{|lll|}
$\begin{array}{l}\text { Type of previous } \\
\text { caesarean section } \\
\mathrm{N}=78\end{array}$ & $\begin{array}{l}\text { Lower uterine } \\
\text { segment } \\
\text { thickness by } \\
\text { USG }(\mathrm{mm}) \\
\text { Mean } \pm \text { SD }\end{array}$ & $\begin{array}{l}\text { Lower uterine } \\
\text { segment } \\
\text { thickness by } \\
\text { calipers }(\mathrm{mm}) \\
\text { Mean } \pm \text { SD }\end{array}$ \\
\hline $\begin{array}{l}\text { Elective caesarean } \\
\mathrm{N}=20\end{array}$ & $2.53 \pm 0.193$ & $2.46 \pm 0.229$ \\
\hline $\begin{array}{l}\text { Emergency caesarean } \\
\mathrm{N}=58\end{array}$ & $2.62 \pm 0.197$ & $2.54 \pm 0.208$ \\
\hline
\end{tabular}

Table 3: Correlation between indication of previous caesarean section and scar thickness.

\begin{tabular}{|c|c|c|c|}
\hline $\begin{array}{l}\text { Indication } \\
\text { of previous } \\
\text { caesarean } \\
\text { section } \\
\mathrm{N}=78\end{array}$ & $\begin{array}{l}\text { Lower } \\
\text { uterine } \\
\text { segment } \\
\text { thickness by } \\
\text { USG }(\mathrm{mm}) \\
\text { Mean } \pm \text { SD }\end{array}$ & $\begin{array}{l}\text { Lower } \\
\text { uterine } \\
\text { segment } \\
\text { thickness } \\
\text { by calipers } \\
\text { (in mm.) } \\
\text { Mean } \pm \text { SD }\end{array}$ & $\begin{array}{l}\text { Statistical } \\
\text { significance }\end{array}$ \\
\hline $\begin{array}{l}\text { Foetal } \\
\text { distress } \\
\mathrm{N}=22\end{array}$ & $2.67 \pm 0.176$ & $2.58 \pm 0.209$ & $\begin{array}{l}t=1.56 \\
P>0.1\end{array}$ \\
\hline $\begin{array}{l}\text { CPD } \\
N=16\end{array}$ & $2.51 \pm 0.21$ & $2.44 \pm 0.247$ & $\begin{array}{l}t=0.86 \\
P>0.1\end{array}$ \\
\hline $\begin{array}{l}\text { Prolonged } \\
\text { labour } \\
\mathrm{N}=15\end{array}$ & $2.65 \pm 0.193$ & $2.56 \pm 0.195$ & $\begin{array}{l}\mathrm{t}=1.28 \\
\mathrm{P}>0.1\end{array}$ \\
\hline $\begin{array}{l}\text { Failed } \\
\text { induction } \\
\mathrm{N}=10\end{array}$ & $2.63 \pm 0.185$ & $2.53 \pm 0.199$ & $\begin{array}{l}t=1.16 \\
P>0.1\end{array}$ \\
\hline $\begin{array}{l}\text { APH } \\
N=5\end{array}$ & $2.46 \pm 0.129$ & $2.43 \pm 0.174$ & $\begin{array}{l}\mathrm{t}=0.3 \\
\mathrm{P}>0.1\end{array}$ \\
\hline $\begin{array}{l}\text { Transverse } \\
\text { lie } \\
\mathrm{N}=2\end{array}$ & $2.58 \pm 0.07$ & $2.55 \pm 0.07$ & $\begin{array}{l}\mathrm{t}=0.42 \\
\mathrm{P}>0.1\end{array}$ \\
\hline $\begin{array}{l}\text { Breech } \\
\mathrm{N}=2\end{array}$ & $2.60 \pm 0$ & $2.54 \pm 0$ & \\
\hline $\begin{array}{l}\text { Elderly } \\
\text { primigravida } \\
\mathrm{N}=1\end{array}$ & 2.24 & 2.12 & \\
\hline $\begin{array}{l}\mathrm{BOH} \\
\mathrm{N}=1\end{array}$ & 2.46 & 2.38 & \\
\hline $\begin{array}{l}\text { Multiple } \\
\text { pregnancy } \\
\mathrm{N}=1\end{array}$ & 2.85 & 2.78 & \\
\hline $\begin{array}{l}\text { Cervical } \\
\text { dystocia } \\
\mathrm{N}=1\end{array}$ & 2.53 & 2.50 & \\
\hline $\begin{array}{l}\text { Eclampsia } \\
\mathrm{N}=1\end{array}$ & 2.20 & 2.08 & \\
\hline $\begin{array}{l}\text { PROM } \\
\mathrm{N}=1\end{array}$ & 2.86 & 2.70 & \\
\hline
\end{tabular}

Table 2 shows correlation between type of previous caesarean section and scar thickness.

Table 3 shows correlation between indication of previous caesarean section and scar thickness.

Table 4 shows correlation between interval between previous caesarean section and present pregnancy and scar thickness.

Table 5 shows correlation between scar thickness with scar tenderness and dehiscence. 
Table 4: Correlation between interval between previous caesarean section and present pregnancy and scar thickness.

\begin{tabular}{|c|c|c|c|c|}
\hline $\begin{array}{l}\text { Interval } \\
\text { between } \\
\text { previous } \\
\text { caesarean } \\
\text { and } \\
\text { present } \\
\text { pregnancy } \\
\text { (years) }\end{array}$ & $\begin{array}{l}\text { No. of } \\
\text { cases } \\
\mathrm{N}=78\end{array}$ & $\begin{array}{l}\text { Scar } \\
\text { thickness } \\
\text { by USG } \\
(\text { mm) } \\
\text { Mean } \pm \text { SD }\end{array}$ & $\begin{array}{l}\text { Scar } \\
\text { thickness } \\
\text { by calipers } \\
(\text { mm) } \\
\text { Mean } \pm \text { SD }\end{array}$ & $\begin{array}{l}\text { Statisti } \\
\text {-cal } \\
\text { signific } \\
\text {-ance }\end{array}$ \\
\hline$<2$ & 7 & $\begin{array}{l}2.27 \pm \\
0.169\end{array}$ & $2.16 \pm 0.2$ & $\begin{array}{l}\mathrm{t}=1.17 \\
\mathrm{P}>0.1\end{array}$ \\
\hline $2-21 / 2$ & 20 & $\begin{array}{l}2.50 \pm \\
0.169\end{array}$ & $2.42 \pm 0.2$ & $\begin{array}{l}\mathrm{t}=1.37 \\
\mathrm{P}>0.1\end{array}$ \\
\hline $2 \frac{1}{2}-3$ & 15 & $\begin{array}{l}2.59 \pm \\
0.124\end{array}$ & $2.52 \pm 0.157$ & $\begin{array}{l}\mathrm{t}=1.37 \\
\mathrm{P}>0.1\end{array}$ \\
\hline$>3$ & 36 & $\begin{array}{l}2.72 \pm \\
0.140\end{array}$ & $2.64 \pm 0.122$ & $\begin{array}{l}\mathrm{t}=2.62 \\
\mathrm{P}<0.05\end{array}$ \\
\hline
\end{tabular}

Table 5: Correlation between scar thickness with scar tenderness and dehiscence.

\begin{tabular}{|c|c|c|c|c|}
\hline $\begin{array}{l}\text { Group of } \\
\text { patients }\end{array}$ & $\begin{array}{l}\text { Scar } \\
\text { tenderness }\end{array}$ & $\begin{array}{l}\text { Scar } \\
\text { dehiscence }\end{array}$ & $\begin{array}{l}\text { Lower } \\
\text { uterine } \\
\text { segment } \\
\text { thickness } \\
\text { by USG } \\
(\mathrm{mm})\end{array}$ & $\begin{array}{l}\text { Lower } \\
\text { uterine } \\
\text { segment } \\
\text { thickness } \\
\text { by } \\
\text { calipers } \\
(\mathrm{mm})\end{array}$ \\
\hline \multirow{6}{*}{$\begin{array}{l}\text { Emergency } \\
\text { caesarean }\end{array}$} & Present & Absent & 2.20 & 2.16 \\
\hline & Present & Present & 2.20 & 2.08 \\
\hline & Present & Present & 2.24 & 2.12 \\
\hline & Present & Absent & 2.20 & 2.16 \\
\hline & Present & Present & 2.20 & 2.08 \\
\hline & Present & Absent & 2.24 & 2.12 \\
\hline \multirow{6}{*}{$\begin{array}{l}\text { Elective } \\
\text { caesarean }\end{array}$} & Present & Present & 2.25 & 2.04 \\
\hline & Absent & Present & 2.34 & 2.16 \\
\hline & Present & Present & 2.20 & 2.08 \\
\hline & Present & Present & 2.24 & 2.02 \\
\hline & Present & Present & 2.31 & 2.18 \\
\hline & Absent & Present & 2.32 & 2.16 \\
\hline
\end{tabular}

\section{DISCUSSION}

In the present study, lower uterine segment thickness measured by ultrasonography correlated well with the thickness measured by vernier calipers at Caesarean section, with a minimum difference of $0.11 \mathrm{~mm}$ in subjects having elective caesarean and of $0.04 \mathrm{~mm}$ in those having emergency caesarean, as shown in Table 1.

Table 2 shows the correlation between type of previous Caesarean section and scar thickness. It reveals no direct correlation.

Table 3 shows the correlation between indication of previous caesarean section and scar thickness. It reveals no direct correlation.
The proportion of cases with thicker lower segment (more than $2.6 \mathrm{~mm}$ ) was higher in cases with longer inter-delivery interval. Table 4 shows the correlation between inter-delivery interval and scar thickness.

Table 5 shows the correlation between scar thickness with scar tenderness and dehiscence. Of 78 cases, 12 cases had defective scars at caesarean section.

All of these 12 cases had lower uterine segment thickness below $2.6 \mathrm{~mm}$ at sonography. The cut-off value of thickness of lower uterine segment was $2.6 \mathrm{~mm}$ as calculated by Receiver Operating Characteristic curve (ROC curve).

If the thickness of lower uterine segment was $2.6 \mathrm{~mm}$ or more, chances of vaginal delivery following trial of labour was high. Lower uterine segment thickness of $<2.6$ $\mathrm{mm}$ was associated with other defects like ballooning of lower segment in 9 out of 12 cases.

\section{CONCLUSIONS}

Prior ultrasonographic assessment of lower uterine segment thickness and scar integrity, can yield a successful trial of labour in properly selected patients with previous Caesarean section in a well-equipped institution under intensive antenatal and intrapartum surveillance. So 'Once a caesarean section, always a caesarean section' policy should be abandoned.

\section{Funding: No funding sources}

Conflict of interest: None declared

Ethical approval: The study was approved by the institutional ethics committee

\section{REFERENCES}

1. Riva HL, Teich JC. Vaginal delivery after caesarean section. Am J Obstet Gynaecol. 1961;181:501-10.

2. Balinga S, Grover B. Ultrasonographic monitoring of scarred lower uterine segment during pregnancy. Obstet Gynaecol Communicat. 1999;2:23-8.

3. Nester Demianczuk, David JS, Hunter. Trial of labour after previous Caesarean section. Prognostic indicators of outcome. Am J Obstet Gynaecol. 1982;142:640.

4. Araki $T$, Inooka $H$. The diagnostic value of ultrasonography with reference to previous caesarean section scars during full term pregnancy. Acta Obstet Gynaecol. 1982;34:738-44.

5. Qureshi B, Inafuku K, Oshima K, Masamoto H, Koj Kanazawa. Ultrasonographic evaluation of lower uterine segment to predict the integrity and quality of Caesarean scar during pregnancy: a prospective study. Tohuku J Exp Med. 1997;183:55-65.

6. Montanari L, Alfei A, Drovanti A, Lepadatu C, Lorenzi D, Facchini D, et al. Transvaginal ultrasonic evaluation of the thickness of the section of the uterine wall in previous caesarean sections. Minerva Gynaecol. 1999;51:107-12. 
7. Asakura H, Nakai A, Ishikawa G, Suzuki S, Araki T. Prediction of uterine dehiscence by measuring lower uterine segment thickness prior to onset of labour; evaluation by transvaginal sonography. J Nippon Med Sci. 2000 Oct;67(5):352-6.
DOI: $10.5455 / 2320-1770 . i j r \operatorname{cog} 20150229$

Cite this article as: Lahiri S, Mondal SC, Pal SR.

Sonographic assessment of lower uterine segment at term with previous caesarean section. Int J Reprod

Contracept Obstet Gynecol 2015;4:164-7. 